\title{
Evaluación de la Degradación Ambiental de Materiales Termoplásticos Empleados en Labores Agrícolas en el Cultivo de Banano en Colombia
}

\author{
José L. Mejía, Herbert Kerguelén, Angélica Gil, Piedad Gañán \\ Grupo de Investigación sobre Nuevos Materiales, Universidad Pontificia Bolivariana
}

\begin{abstract}
Resumen: La producción de banano es una dinámica actividad económica en Colombia, sin embargo durante las labores agrícolas, las plantas deben ser unidas unas a otras a través de cuerdas. Esta prácticas contribuye a solventar el peso de los frutos antes de la cosecha. Cuerdas de polipropileno son utilizadas para este fin. Sin embargo, esta práctica genera problemas ambientales en las regiones de cultivo, haciendo necesario identificar materiales biodegradables alternativos. En este estudio, muestras de polipropileno, mezclas de polipropileno con almidón, así como un biopolímero conocido como Mater$B i$ y éste combinado con PP fueron analizados. Su estabilidad fue evaluada al ser expuestas a las condiciones medioambientales de la región tropical de Urabá (Colombia). Los cambios en la estructura de los materiales expuestos fueron analizados utilizando calorimetría diferencial de barrido (DSC) y espectroscopía infrarroja FTIR. Pequeñas diferencias en el comportamiento mecánico y físico de las muestras expuestas fueron observadas, y pese a que la adición tanto del almidón como del biopolímero reducen el comportamiento a tracción del polipropileno, estas mezclas pueden ser útiles para esta aplicación.
\end{abstract}

Palabras-claves: Almidón, aplicaciones agrícolas, biopolímeros, comportamiento a tracción, exposición ambiental, polipropileno.

\section{Environmental Degradation of Thermoplastic Materials used in Banana Agricultural Labors in Colombia}

Abstract: Banana fruit production is a dynamic economical sector in Colombia. However during the agricultural labor, banana plants need to be tied to each other using strings. This practice helps to support the fruit weight before the harvesting. Polypropylene strings are used in this application. This practice is becoming an environmental issue in cultivation areas, and it is necessary to identify biodegradable alternative materials. In this study, samples of polypropylene, polypropylene/starch blend and a commercial biopolymer, referred to as Mater-Bi and PP/ Mater-Bi, were analyzed. The stability of these materials in tropical regions such as Uraba (Colombia) was evaluated. The changes in the material structure and properties were analyzed by differential scanning calorimetry (DSC) and FTIR spectroscopy. Slight variations of mechanical and physical behavior of exposed samples were observed. Even though starch and biopolymer reduced the tensile behavior of polypropylene blends, these blends can be used for this application.

Keywords: Starch, agricultural applications, biopolymer, tensile behavior, environmental weathering, polypropylene.

\section{Introducción}

En Colombia existen más de 40.000 hectáreas dedicadas a la producción de banano, ubicándolo en los primeros renglones de exportación en el ámbito mundial. Una de las principales regiones de cultivo en este país corresponde a la zona de Urabá (Antioquia), donde se concentra más del $70 \%$ del área cultivada del mismo. Dentro de las labores agrícolas requeridas, el apuntalamiento es clave. Este proceso también conocido como sostén o amarre de las plantas, se realiza como respuesta a la necesidad de solventar el peso de la misma, debido al crecimiento de los racimos y a la par contribuye con su protección frente a las fuertes condiciones de carga de viento que se presentan en las regiones tropicales ${ }^{[1]}$. Uno de los materiales plásticos más empleados para esta actividad es el polipropileno, pues ofrece diferen- tes ventajas tanto a nivel técnico como económico, entre las que se encuentran: bajo costo y densidad $\left(0,90 \mathrm{~g} / \mathrm{cm}^{3}\right)$ y apropiada resistencia mecánica a tracción. La actual labor de apuntalamiento o sostén que tiende lugar en algunas regiones de Colombia se realiza durante el tiempo comprendido entre la inclinación de la bacota y la cosecha, con el fin de evitar su caída durante el proceso de crecimiento del racimo y que tarda entre 13 y 14 semanas aproximadamente. Una vez la fruta tiene la edad adecuada para la cosecha, se retiran las cuerdas de las plantas y se procede a retirar el racimo. En muchos casos estos materiales son arrojados al suelo sin mayor control en su disposición final, generando serios problemas de contaminación que pueden afectar los procesos biológicos y que alteran características del suelo como su permeabilidad, afectando así el rendimiento del cultivo. Otras veces, son recogidos para ser 
posteriormente reciclados. Sin embargo, en este último caso es necesario realizar una labor de limpieza que permita remover impurezas de su superficie y que incluyen fungicidas que han sido aplicados a la planta, situación que incrementa los costos del proceso sumado al inferior desempeño técnico del material reciclado obtenido ${ }^{[2]}$. Una potencial alternativa a ambas prácticas es elaborar dichos elementos en poliolefinas con aditivos que fomenten su incorporación al suelo, como ocurre con el almidón por ejemplo ${ }^{[3]} \mathrm{o}$ incluso emplear polímeros biodegradables ${ }^{[2,4]}$. En este trabajo se ha evaluado el comportamiento físico y mecánico de muestras de polipropileno aditivado así como un biopolímero conocido como Mater-Bi que puedan ser empleados durante las labores de apuntalamiento o sostén de plantas en regiones tropicales como el Urabá Antioqueño en Colombia. Se presenta un estudio comparativo de las variaciones en algunas de las propiedades físicas (densidad, índice de fluidez) y mecánicas (resistencia a la tracción) de los posibles polímeros que podrían sustituir al polipropileno actualmente utilizado. La calorimetría diferencial de barrido (DSC) así como la espectroscopía han sido empleadas con miras a establecer los posibles efectos degradativos que se puedan presentar en la estructura de los diferentes materiales.

\section{Desarrollo Experimental}

\section{Materiales}

En el caso del polipropileno, se utilizó la referencia de Propilco 03 H82 suministrado por la empresa Plassol (Colombia). Como aditivos se emplearon almidón suministrado por Industrias del Maíz (Colombia), material conformado por amilasa y amilopectina con una densidad de $1,6 \mathrm{~g} / \mathrm{cm}^{3}$ y Mater-Bi NFOIU (Novamont, USA) con una densidad de $1,3 \mathrm{~g} / \mathrm{cm}^{3}$, que corresponde a un copolímero de almidón y un poliéster. Este último además de ser utilizado como aditivo, se empleó sin mezclar.

\section{Elaboración de las muestras y exposición ambiental}

Se elaboraron placas de aproximadamente $3,5 \mathrm{~mm}$ de espesor de los diferentes materiales a estudiar, utilizando un equipo de doble rodillos para realizar la mezcla en una proporción y temperatura determinada, y posteriormente se sometieron a un proceso de compresión en caliente. En la Tabla 1 se presentan las combinaciones de materiales, así como las condiciones de transformación de los materiales utilizados y se indican las abreviaturas con las cuales se seguirán nombrando en los demás ítems.

Las placas de los diferentes materiales se cortaron de forma rectangular y luego fueron perforadas en los extremos para facilitar la operación amarre, esta forma de exposición permite simular las condiciones de uso reales, como se muestra en la Figura 1. El tamaño de las muestras corresponden a $35,3 \times 205,0 \times 3,5 \mathrm{~mm}$. El amarre superior en la planta se hizo entre la tercera y cuarta hoja con un anclaje de dos
Tabla 1. Materiales desarrollados y sus condiciones de transformación.

\begin{tabular}{llcc}
\hline & & \multicolumn{2}{c}{ Temperatura $\left({ }^{\circ} \mathbf{C}\right)$} \\
\cline { 3 - 4 } \multicolumn{1}{c}{ Material } & Sigla & Mezclado & Compresión \\
\hline PP sin aditivarr & PP & 180 & 180 \\
PP + almidón $\left(20^{*}\right)$ & PPA20 & 180 & 180 \\
PP + Mater-Bi (30*) & PPM30 & 160 & 160 \\
PP + Mater-Bi (50*) & PPM50 & 160 & 160 \\
Mater-Bi sin aditivarr & M & 160 & 160 \\
\hline
\end{tabular}

* Porcentaje en peso del aditivo respecto a la masa total del material.

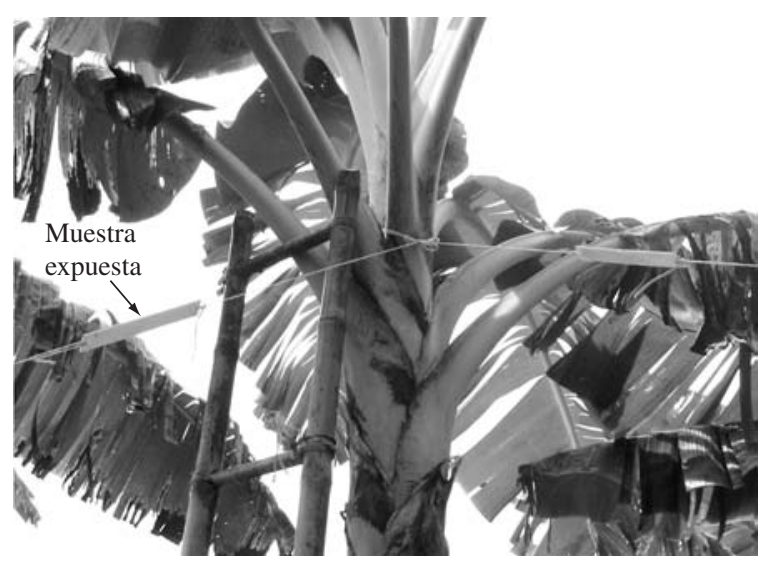

Figura 1. Montaje de las muestras expuestas a las condiciones de Urabá.

vientos, con ángulos de 45 grados y a una altura de $70 \mathrm{~cm}$ con relación al suelo en plantas vecinas que sirven de soporte ${ }^{[1]}$.

Las muestras fueron ubicadas en una plantación de la región de Turbo (Urabá Antioqueño) con el apoyo de la Fundación Social Corbanacol, por un período entre 7 y 14 semanas. El tiempo máximo de exposición se determinó de acuerdo a la duración de la cosecha, que es el tiempo que generalmente permanece las cuerdas en uso. Las condiciones ambientales corresponden a una humedad relativa promedio de $95 \%$ y una temperatura promedio de $30{ }^{\circ} \mathrm{C}$.

\section{Caracterización de los materiales}

La caracterización de los materiales antes y tras la exposición corresponde a la determinación de la densidad mediante la norma ASTM D792, índice de fluidez (MFI) con la norma ASTM D1238 y el comportamiento a tracción con la norma ASTM D638. El equipo empleado para el ensayo de tracción es una máquina universal marca Instron modelo 5582. Los ensayos fueron realizados a una velocidad de $5,08 \mathrm{~mm} / \mathrm{min}$. Para cada material se analizaron alrededor de cinco probetas.

En cuanto a los ensayos de calorimetría diferencial de barrido, se realizaron en un equipo DSC 2920 de la marca TA Instruments en atmósfera de nitrógeno con un flujo de $50 \mathrm{ml} / \mathrm{min}$, empleando ciclos de calentamiento de $10{ }^{\circ} \mathrm{C} / \mathrm{min}$ hasta una temperatura máxima de $200{ }^{\circ} \mathrm{C}$, que fue sostenida por 5 minutos, para luego enfriar a una velocidad controlada $10{ }^{\circ} \mathrm{C} / \mathrm{min}$. Posibles cambios sobre la estructura química de las muestras fueron analizados empleado un equipo de espectroscopia infrarroja por transformada de Fourier, FTIR marca 
Perkin Elmer PC1600. La resolución de los espectros fue de $4 \mathrm{~cm}^{-1}$ y fueron realizados 20 barridos por muestra.

\section{Resultados y Análisis}

De acuerdo con los resultados observados en la Figura 2, se aprecia como era de suponerse, que la densidad de las mezclas sin exponer incrementará por efecto de ambos tipos de aditivos. Sin embargo, en todos los materiales analizados no se presentaron importantes cambios en los valores de densidad antes y tras la exposición. De acuerdo con lo reportado por autores como Gulmine et al. ${ }^{[5]}$, la densidad de las poliolefinas tiende a incrementar por efecto de la degradación debida a la exposición ambiental, posiblemente en este caso el nivel de degradación registrado sea bajo al encontrarse en una fase inicial debido al corto tiempo de exposición.

En el caso del índice de fluidez, tanto las muestras con aditivos como la de biopolímero mostraron una tendencia a incrementar este valor conforme transcurre el tiempo de exposición siendo en mayor medida en el caso del biopolímero y la muestra PPM50 como se aprecia en la Figura 3. Esta

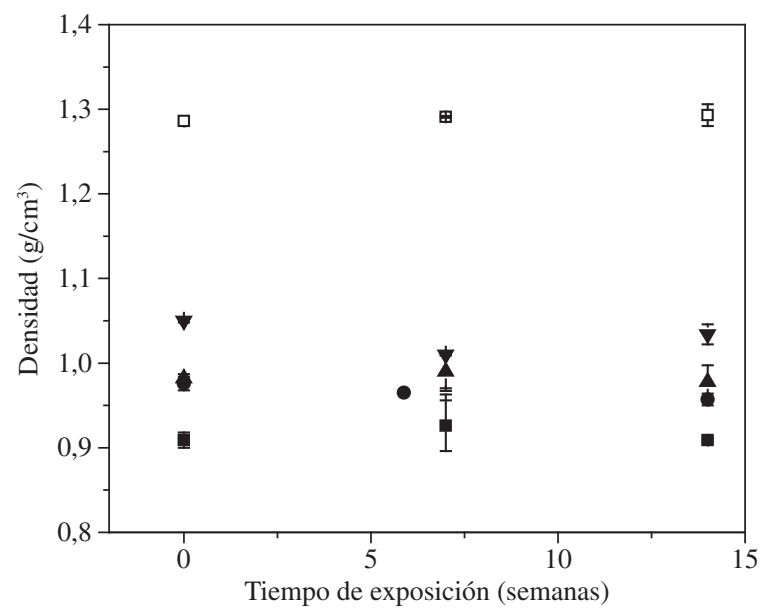

Figura 2. Variación de la densidad de muestras sometidas a la exposición

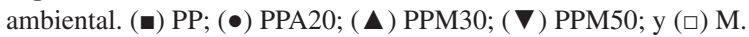

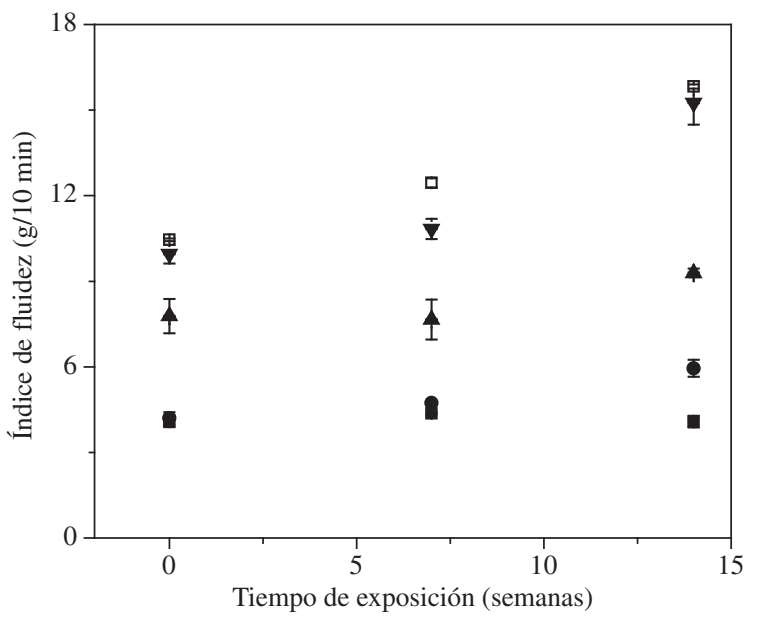

Figura 3. Variación del índice de fluidez de muestras sometidas a la exposi-

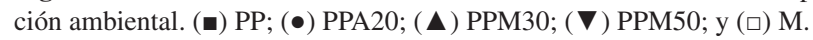

circunstancia probablemente se encuentre asociada con el comienzo de procesos degradativos, entre lo cuales uno de los más importantes corresponde a la disminución en el peso molecular de las cadenas al final de la exposición ambiental de los polímeros debido a la influencia de los aditivos empleados ${ }^{[6]}$. Para la muestra de PP, los cambios en los valores del índice de fluidez al transcurrir las 14 semanas no registran variaciones significativas, lo cual se relacionaría de nuevo a la corta exposición ambiental, que impide que se presenten cambios importantes en las zonas amorfas de este polímero ${ }^{[7]}$.

Al comparar el comportamiento mecánico de las muestras sin exposición, como se registra en la Figura 4, la presencia tanto del almidón como del biopolímero a diferentes porcentajes reducen la resistencia máxima del polipropileno, debido posiblemente a una baja adhesión interfacial, que conduce a una ruptura mecánica en la interfase de la mezcla, como se ha documentado en otros sistemas ${ }^{[5,8]}$. Sin embargo, pese a esta circunstancia, las muestras aditivadas están en capacidad de soportar la condición de trabajo y exposición durante el apuntalamiento, muestra de ello es la ausencia volcamientos o pérdida de racimos durante el tiempo que transcurrió el ensayo, registrando también un mejor desempeño mecánico que el biopolímero sin aditivar.

En la Figura 4 se observa que la resistencia máxima de los diferentes materiales no experimenta una sensible variación por efecto de la exposición medioambiental, esta circunstancia puede estar asociada a diferentes fenómenos que incluyen un bajo nivel de degradación debido al tiempo que tarda la exposición, y que se aprecia particularmente en el caso del PP, así como mejoras al interior de las diferentes mezclas debidas a la degradación inicial de ambos componentes, y que pueden afectar la polaridad de los componentes y por ende la compatibilidad al interior de la mezcla, reflejándose entonces en el desempeño mecánico de las mezclas. Resultados comparables en este sentido fueron observados por otros autores en sistemas de polipropileno cargado con aditivos celulósicos como las fibras de palma tras ser expuestas a diferentes condiciones ambientales ${ }^{[9]}$.

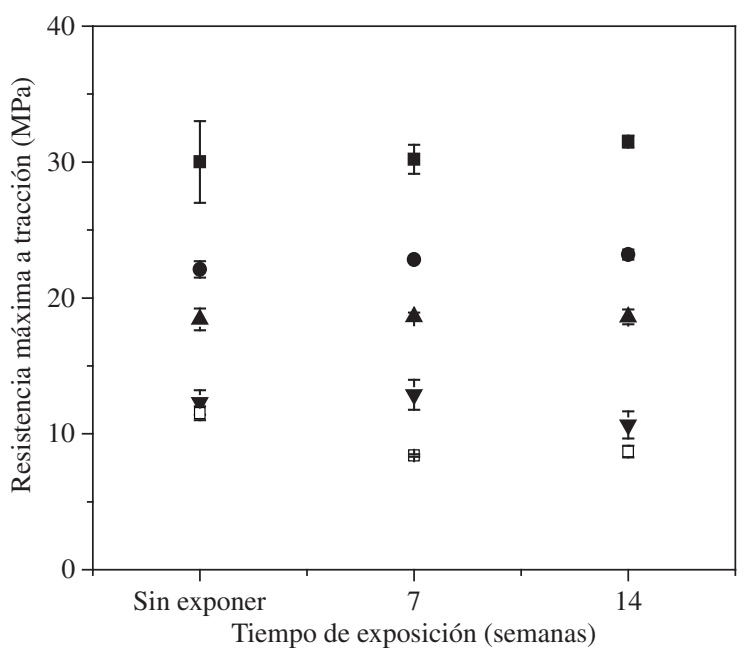

Figura 4. Variación de la resistencia máxima a tracción de muestras so-

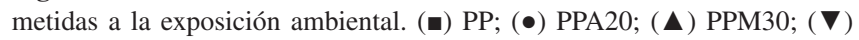
PPM50; y (口) M. 
En la Tabla 2 se aprecia que las temperaturas de fusión de las diferentes muestras evaluadas mediante calorimetría diferencial de barrido tras la exposición ambiental, experimentan un ligero incremento, resultados que podría estar asociado a variaciones en la cristalinidad del polipropileno debidas posiblemente a la reorganizan de las cadenas motivados por las posibles variaciones en el peso molecular ${ }^{[6,10]}$. Pero estos reducidos cambios nuevamente sugieren que el grado de degradación alcanzado por todas las muestras es bajo.

De otro lado, en las Figuras 5 y 6 se presentan los espectros infrarrojos de las muestras PPA20 y M. En el primer caso, se aprecia que aún la muestra sin exponer tiene una banda de vibra-

Tabla 2. Variación de la temperatura de fusión evaluada mediante calorimetría diferencial de barrido de muestras sometidas a la exposición ambiental.

Temperatura de fusión $\left({ }^{\circ} \mathrm{C}\right)$

\begin{tabular}{lcc} 
Material & Sin exponer & Tras 14 semanas de exposición \\
\hline PP & $166,9 \pm 0,3$ & $167,3 \pm 0,4$ \\
PPA20 & $165,6 \pm 0,2$ & $166,0 \pm 0,1$ \\
PPM30 & $165,5 \pm 0,2$ & $166,0 \pm 0,2$ \\
PPM50 & $163,7 \pm 0,2$ & $164,4 \pm 0,2$ \\
\hline
\end{tabular}

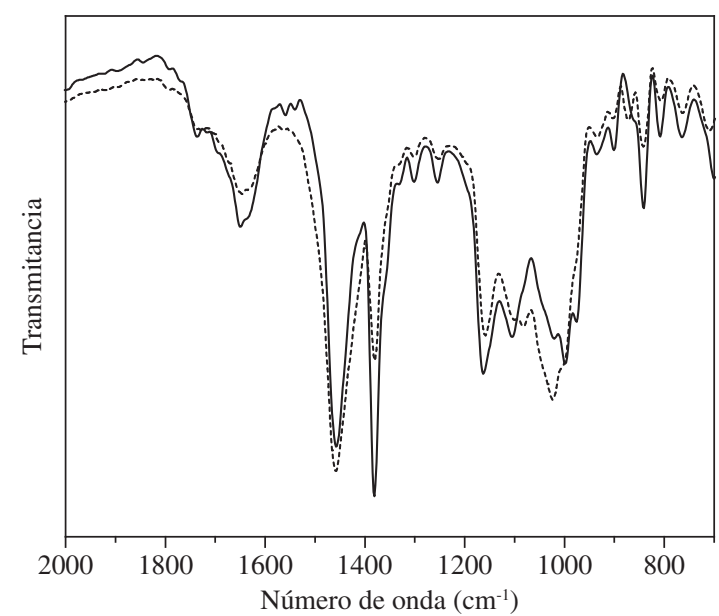

Figura 5. Espectros infrarrojos de muestras de PPA20: (-) sin exponer; y (- -) tras la exposición ambiental.

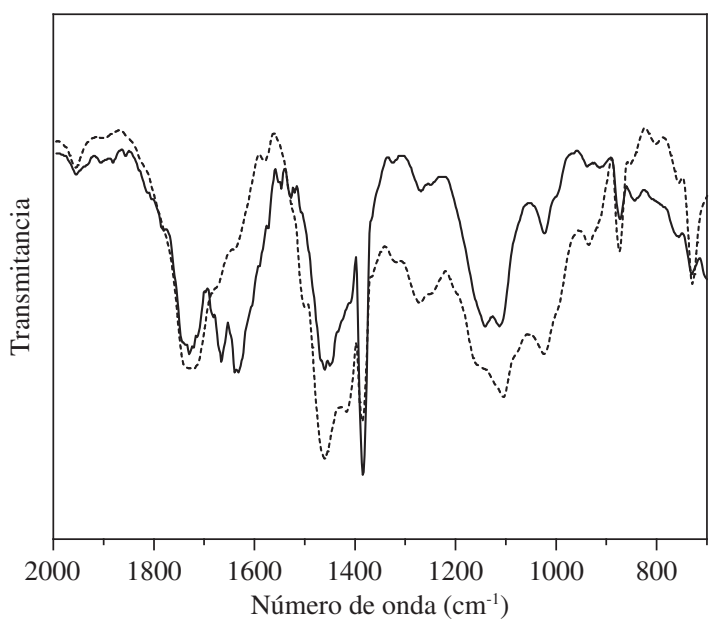

Figura 6. Espectros infrarrojos de muestras de M. (-) sin exponer; y (- -) tras la exposición ambiental. ción en la región de $1730 \mathrm{~cm}^{-1}$, la cual se encuentra relacionado posiblemente con aditivos presentes en el polipropileno, razón por la cual es difícil establecer la formación de nuevas vibraciones de este tipo de grupos debidas a la exposición ambiental del polímero. Lo que si se hace evidente es la transformación que tienen lugar en el almidón, pues se observan cambios significativos en la región entre 1200 y $900 \mathrm{~cm}^{-1}$, los cuales están relacionadas con la degradación del almidón y que se manifiestan mediante la reducción de bandas como 1000 y $976 \mathrm{~cm}^{-1}$, así como el surgimiento de otras como $1076 \mathrm{~cm}^{-1}$, y que guardan relación con la degradación de este aditivo ${ }^{[11]}$. En el caso de las muestras de biopolímeros, en la Figura 6 se presentan ligeras variaciones alrededor de la vibración a $1780 \mathrm{~cm}^{-1}$, relacionados con el inicio de procesos degradativos en la porción sintética del polímero. Estos resultados se encuentran en concordancia por lo reportado por otros autores como Massardier-Nageotte y otros en sus estudios sobre degradación de films de poliésteres biodegradables ${ }^{[12]}$. Otros cambios más significativos se presentan en la región entre $1700-1600 \mathrm{~cm}^{-1}$, y que pueden guardar relación con las variaciones en la estructura cristalina del polímero y que afectan en este caso su índice de fluidez tal como se ha comentado antes. Es posible sin embargo, que los cambios apreciados tengan lugar principalmente a nivel superficial debido a la forma en que las muestras fueron expuestas, lo cual explicaría porque la variación en las demás propiedades y características analizadas es baja.

\section{Conclusiones}

En este trabajo muestras tanto de polipropileno como sus mezclas con almidón y biopolímero así como el biopolímero puro fueron sometidas a las condiciones de exposición ambiental y de carga mecánica requeridas por una cuerda de soporte para plantas de banano en la región de Urabá. Los resultados indican que la condición de exposición ambiental evaluada induce a un bajo nivel de degradación en los diferentes materiales al tener lugar esencialmente en la superficie, lo cual explicaría las variaciones observadas en la estructura química a través de la espectroscopía infrarroja y la baja reducción en las demás propiedades y características.

Pese a que tanto el almidón como el biopolímero al ser utilizados como aditivos reducen el desempeño mecánico del polipropileno, estas mezclas pueden ser viables de utilizar durante esta aplicación con miras a incrementar la biodegradabilidad del sistema debido a la baja reducción en resistencia mecánica que experimentan durante la exposición ambiental.

\section{Agradecimientos}

Los autores expresan su agradecimiento al Instituto Colombiano para el Desarrollo de la Ciencia y la Tecnología "Francisco José de Caldas" COLCIENCIAS por su apoyo financiero al desarrollo del proyecto Cod. 12100814702. Contrato 442-2003, del cual hacen parte los resultados comentados en este trabajo. 


\section{Referencias Bibliográficas}

1. Corbanacol, "Manual de tecnificación del cultivo del plátano", Fundación Social Banacol, Medellín (2001).

2. Ren, X. - Journal of Cleaner Production, 11, p.27 (2003).

3. Ramis, X.; Cadenato, A.; Salla, J.; Morancho, J; Vallés, A.; Contat, L. \& Ribes, A. - Polymer degradation and stability, 86, p.483 (2004).

4. Contact-Rodrigo, L.; Ribes-Greus, A. \& Díaz-Calleja, R. - Journal of Applied Polymer Science, 82, p.2174 (2001).

5. Gulmine, J.; Janissek, P.; Heise, H. \& Akcelrud, L. - Polymer degradation and stability, 79, p.385 (2003).

6. Pedroso, A. \& Rosa, D. - Carbohydrate Polymers, 59, p.1 (2005).
7. Elvira, M.; Tiemblo, P. \& Gómez-Elvira, J. - Polymer Degradation and Stability, 83, p.509 (2004).

8. Azhari, C. \& Wong, S. - Pakistan Journal of Biological Sciences, 4, p.693 (2001).

9. Abu-Sharkh, B. \& Hamid, H. - Polymer Degradation and Stability, 85, p.967 (2004).

10. Morancho, J.; Ramis, X.; Fernández, X.; Cadenato, A.; Salla, J.; Vallés A.;Contat, L. \& Ribes. A. - Polymer Degradation and Stability, 91, p.44 (2006).

11. Nikonenko, N.; Buslov, D.; Sushko, N. \& Zhbankov, R. - Journal of Molecular Structure, 752, p.20 (2005).

12. Massardier-Nageotte, V.; Pestre, C.; Cruard-Prade, T. \& Bayard, R. - Polymer Degradation and Stability, 91, p. 620 (2006).

Enviado: 06/12/06

Reenviado: 13/03/07

Aceito: $26 / 03 / 07$ 